\title{
Percutaneous transluminal angioplasty with carbon dioxide for peripheral arterial disease after kidney transplantation: a case report with literature review
}

\author{
Masataka Banshodani ${ }^{*}$, Seiji Marubayashi ${ }^{1}$, Sadanori Shintaku' ${ }^{1}$, Tomoyasu Sato², Misaki Moriishi ${ }^{1}$, \\ Shinichiro Tsuchiya ${ }^{1}$, Hideki Ohdan ${ }^{3}$ and Hideki Kawanishi ${ }^{1}$
}

\begin{abstract}
Background: Arteriosclerosis may progress and lead to peripheral arterial disease (PAD) during the waiting period until kidney transplantation in end-stage kidney disease (ESKD) patients. Additionally, contrast-induced nephropathy (CIN) of a kidney allograft after the examination and treatment for PAD is problematic. Here, we report the case of a kidney transplant recipient with PAD in the lower extremities who underwent percutaneous transluminal angioplasty (PTA) with carbon dioxide to prevent CIN incidence.

Case presentation: A 57-year-old woman underwent a cadaveric kidney transplant when she was 49 years old. Immunosuppression was maintained with tacrolimus, methylprednisolone, and mycophenolate mofetil. Her posttransplant course was uneventful, and serum creatinine level was maintained at 1.1-1.3 mg/dL. Intermittent claudication of the lower legs began 3 years after transplantation. Under saline intravenous rehydration, computed tomographic angiographies were performed, and the patient was diagnosed with PAD in the bilateral lower extremities. Total PTA was performed thrice for PAD in the lower extremities via a combination of carbon dioxide and iodinated contrast medium to prevent CIN incidence at 3, 4, and 7 years after kidney transplantation. The patient's recoveries were uneventful. One year later, the serum creatinine level was maintained at 0.9-1.1 mg/dL, and since then, the patient has shown no evidence of recurrence.
\end{abstract}

Conclusions: In a kidney transplant recipient with PAD, PTA with carbon dioxide was effective to minimize the volumes of iodinated contrast medium and prevent CIN incidence.

Keywords: Arteriosclerosis obliterans, Carbon dioxide, Contrast-induced nephropathy, Kidney transplantation, Percutaneous transluminal angioplasty, Peripheral arterial disease

\footnotetext{
* Correspondence: m-banshodani@tsuchiya-hp.jp

'Department of Artificial Organs, Akane-Foundation, Tsuchiya General

Hospital, 3-30, Nakajimacho, Naka-ku, Hiroshima 730-8655, Japan

Full list of author information is available at the end of the article
}

(c) The Author(s). 2020 Open Access This article is licensed under a Creative Commons Attribution 4.0 International License, which permits use, sharing, adaptation, distribution and reproduction in any medium or format, as long as you give appropriate credit to the original author(s) and the source, provide a link to the Creative Commons licence, and indicate if changes were made. The images or other third party material in this article are included in the article's Creative Commons licence, unless indicated otherwise in a credit line to the material. If material is not included in the article's Creative Commons licence and your intended use is not permitted by statutory regulation or exceeds the permitted use, you will need to obtain permission directly from the copyright holder. To view a copy of this licence, visit http://creativecommons.org/licenses/by/4.0/ The Creative Commons Public Domain Dedication waiver (http://creativecommons.org/publicdomain/zero/1.0/) applies to the data made available in this article, unless otherwise stated in a credit line to the data. 


\section{Background}

Arteriosclerosis may progress and lead to peripheral arterial disease (PAD) during the waiting period until kidney transplantation in end-stage kidney disease (ESKD) patients, and the symptoms of PAD may be noticed after kidney transplantation. Moreover, PAD is associated with cardiovascular events and mortality [1-4].

Kidney transplant recipients are highly susceptible to chronic kidney disease (CKD) [5]. CKD is the most common risk factor for contrast-induced nephropathy (CIN) [6]. Therefore, CIN due to iodinated contrast medium after the examination and treatment for PAD is problematic for kidney transplant patients $[7,8]$.

Here, we report the case of a kidney transplant recipient with PAD in the lower extremities who underwent percutaneous transluminal angioplasty (PTA) with carbon dioxide to prevent CIN incidence. Written informed consent for the use of personal information was obtained from the patient.

\section{Case presentation}

A 57-year-old woman received a cadaveric kidney transplant (left kidney graft) for ESKD due to lupus nephritis when she was 49 years old. Immunosuppression was induced with tacrolimus, methylprednisolone, mycophenolate mofetil, and basiliximab for 20 days and maintained with tacrolimus (1.5 mg/day), methylprednisolone ( $4 \mathrm{mg}$ / day), and mycophenolate mofetil (750 mg/day). Her post-transplant course was uneventful, and serum creatinine level and body weight were maintained within the ranges of $1.1-1.3 \mathrm{mg} / \mathrm{dL}$ and $46-49 \mathrm{~kg}$, respectively. However, 3 years post-transplantation, intermittent claudication of the lower extremities was observed. Under saline intravenous rehydration before and after (each $500 \mathrm{~mL}$ ), computed tomographic angiographies (CTAs) were performed thrice with iohexol with an iodine concentration of $300 \mathrm{mg} / \mathrm{ml}$ (Ioverin 300; Teva Takeda Pharma Ltd., Nagoya, Japan): the amount of the administered iodinated contrast medium was $100 \mathrm{~mL}$ for each CTA, and the patient was diagnosed with PAD in the bilateral lower extremities at 3, 4, and 7 years after kidney transplantation (Fig. $1 \mathrm{a}-\mathrm{c}$ ). PTAs were performed thrice for PAD in the lower extremities via a combination of carbon dioxide (SODA CARTRIDGE; NIPPON TANSAN GAS CO., LTD., Tochigi, Japan; $200 \mathrm{~mL}$ for each PTA) for all lesions and iodixanol with an iodine concentration of $320 \mathrm{mg} / \mathrm{ml}$ (Visipaque 320; Daiichi Sankyo, Tokyo, Japan) for only below-the-knee arterial stenotic lesions to prevent the incidence of CIN at 3, 4, and 7 years after kidney transplantation (Fig. 1d-f); the amounts of the administered iodinated contrast medium at these PTAs were $15 \mathrm{~mL}, 30 \mathrm{~mL}$, and $20 \mathrm{~mL}$, respectively. We used the iodinated contrast medium in addition to carbon dioxide for below-the-knee arterial stenotic lesions in the patient due to the defocused image of the below-the-knee arterial stenotic lesions produced by carbon dioxide. The patient had leg pain for 2 weeks only after the first PTA but did not have any major complications after these PTAs. One year later, the serum creatinine level was maintained at 0.9-1.1 $\mathrm{mg} / \mathrm{dL}$ (Fig. 2), and the patient has shown no evidence of recurrence.

\section{Discussion}

CIN is a common cause of hospital-acquired acute kidney injury [9]. Neyra et al. reported that CIN was associated with adverse in-hospital and long-term outcomes in both CKD and non-CKD patients [10]. Moreover, several studies demonstrated that CIN was associated with the development of CKD [11-13].

Kidney transplant recipients require calcineurin inhibitors for immunosuppressive treatment to prevent graft rejection, but kidney allograft function is mostly reduced as a result of this usage due to the nephrotoxicity of the calcineurin inhibitor and non-immunosuppressive burden [5]. Ahuja et al. reported that $21.2 \%$ of kidney transplant patients had CIN [7]. In a report by Light et al., CIN was more common and severe in patients with kidney allograft dysfunction than those without [14].

Cigarroa et al. advocated a guideline to limit iodinated contrast medium to significantly reduce the incidence of CIN: $5 \mathrm{~mL}$ of iodinated contrast medium per kilogram body weight/serum creatinine $(\mathrm{mg} / \mathrm{dL})$ with a maximum dose of $300 \mathrm{~mL}$ [15]. According to a cohort study by Gruberg et al. regarding CKD patients (baseline serum creatinine $\geq 1.8 \mathrm{mg} / \mathrm{dL}$ ) undergoing percutaneous coronary intervention (PCI), the amount of iodinated contrast medium was significantly higher in patients who developed CIN compared with those who did not develop CIN $(261 \pm 148 \mathrm{~mL}$ vs $214 \pm 98 \mathrm{~mL})$ [16]. In a study regarding CKD patients (serum creatinine $>1.2 \mathrm{mg} / \mathrm{dL}$ and/or estimated creatinine clearance $(\mathrm{CrCl})<70 \mathrm{~mL} /$ min) undergoing elective coronary and/or peripheral angiography and/or angioplasty, Briguori et al. identified a volume of iodinated contrast medium $\geq 140 \mathrm{~mL}$ as the best cutoff value to predict the occurrence of CIN (sensitivity $89 \%$, specificity 55\%) [17]. In a report by Laskey et al. regarding patients undergoing $\mathrm{PCI}$, the amount of iodinated contrast medium tended to be higher in patients who developed CIN compared with those who did not develop CIN $(255 \pm 124 \mathrm{~mL}$ vs $224 \pm 112 \mathrm{~mL})$, and the authors concluded that a ratio of the volume of iodinated contrast medium to the $\mathrm{CrCl}(\mathrm{V} / \mathrm{CrCl})>3.7$ was a significant and independent predictor of developing CIN after PCI in the unselected patient population including both CKD and non-CKD patients [18]. Nevertheless, clinicians should minimize the volumes of 


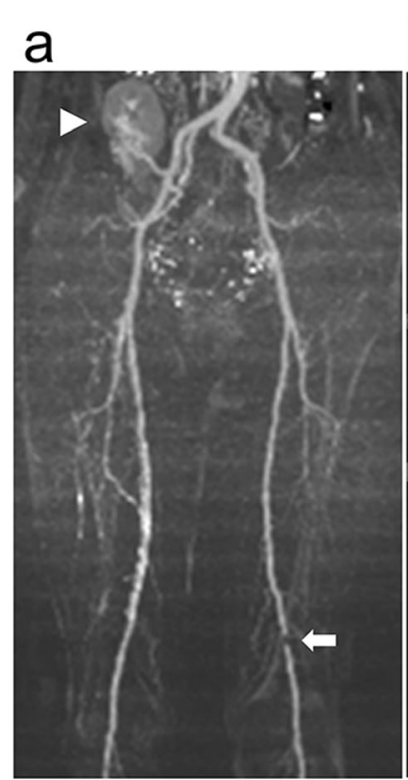

b c

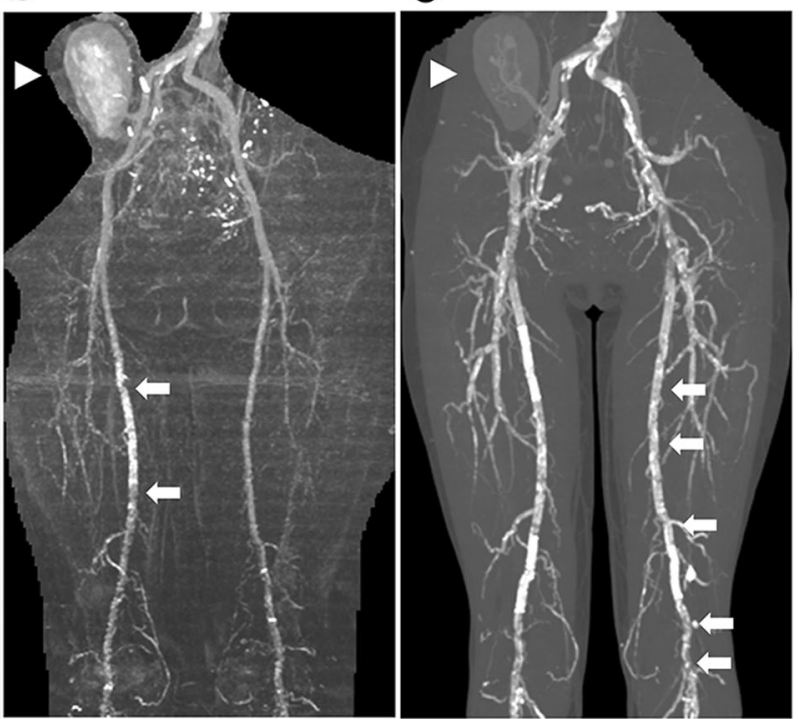

d

e

f
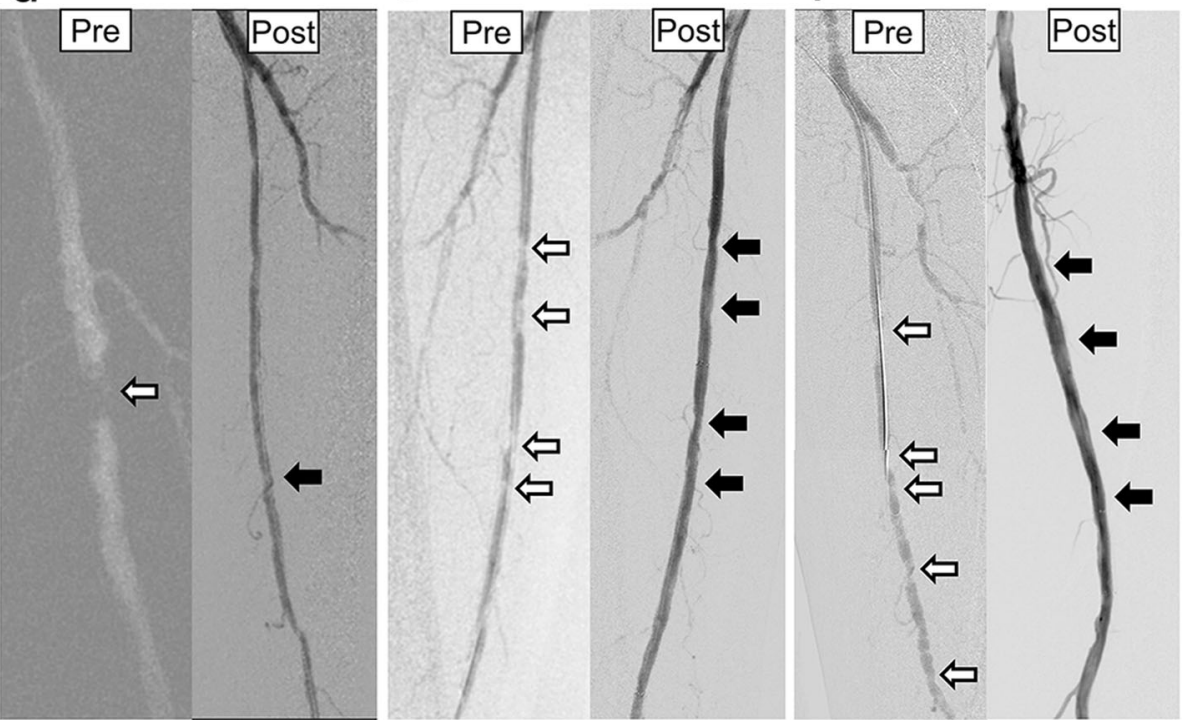

Fig. 1 Computed tomographic angiography (CTA) and percutaneous transluminal angioplasty (PTA) for peripheral arterial disease in a kidney transplant recipient. CTA revealing arterial stenotic lesions in the left or right femoral artery (white arrows) and the kidney graft in the right retroperitoneal space (white arrow heads): a first, $\mathbf{b}$ second, and $\mathbf{c}$ third CTA. Angiography with carbon dioxide or iodinated contrast medium revealing stenotic lesions before and after PTA: $\mathbf{d}$ first PTA by carbon dioxide, e second PTA by iodinated contrast medium, and $\mathbf{f}$ third PTA by carbon dioxide at pre-PTA and iodinated contrast medium at post-PTA (pre-PTA, white arrows; post-PTA, black arrows)

iodinated contrast medium at the time of examination and treatment for PAD in kidney transplant recipients.

Carbon dioxide is safely transported to the lungs, where it is eliminated by exhalation as a result of its 20fold greater solubility in blood compared with oxygen and its ability to combine with blood buffers [19]. In guidelines on the use of iodinated contrast medium in patients with kidney disease which were developed in collaboration with the Japanese Society of Nephrology, the Japan Radiological Society, and the Japanese
Circulation Society [20], the use of carbon dioxide was not mentioned to prevent CIN in patients with kidney disease. However, carbon dioxide has been used as an intra-arterial contrast medium during angiographyguided interventions in patients with PAD and kidney dysfunction because of its status as a non-allergen, affordability, and the absence of nephrotoxicity [21]. Moreover, the quality of angiography with carbon dioxide has improved due to the development of radiographic technology [22]. 


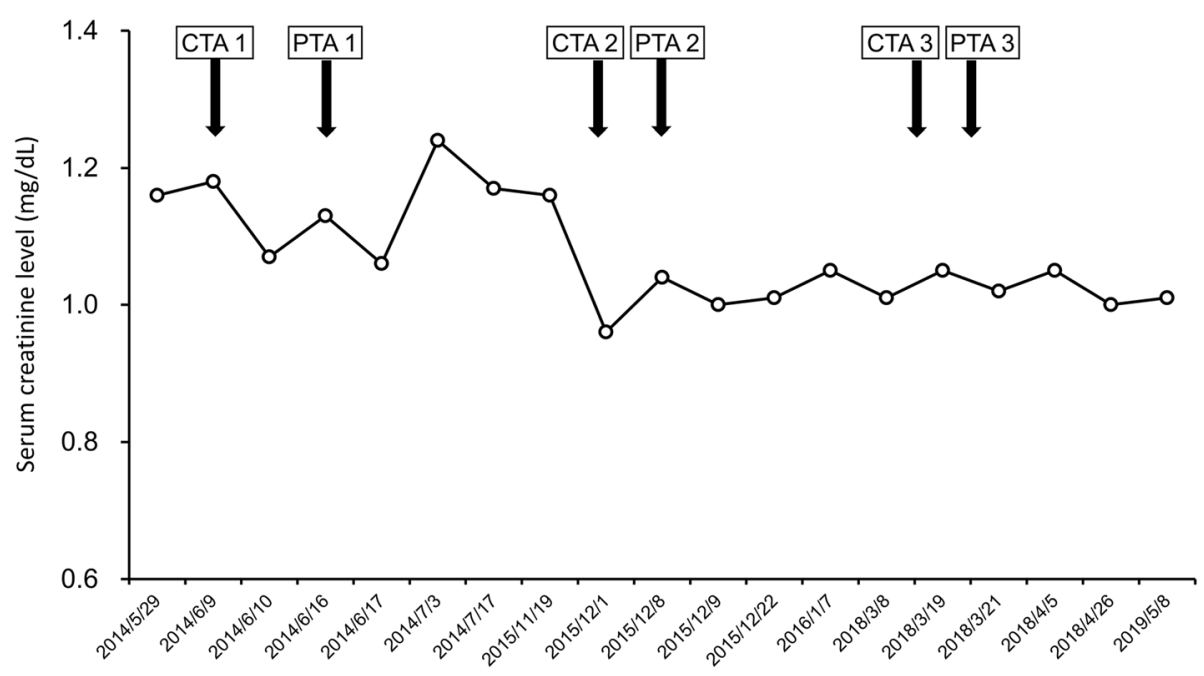

Fig. 2 Transition of serum creatinine level after computed tomographic angiography and percutaneous transluminal angioplasty for peripheral arterial disease in a kidney transplant recipient. CTA, computed tomographic angiography; PTA, percutaneous transluminal angioplasty

In a prospective study for patients with CKD undergoing peripheral angioplasty procedures, the incidence of CIN was significantly higher in the iodinated contrast medium group (29\%) compared with the carbon dioxide group (14\%) [23]. In a single-center study of carbon dioxide angiography for guiding kidney-related interventions in CKD (serum creatinine $\geq 3.0 \mathrm{mg} / \mathrm{dL}$ ) patients with Takayasu arteritis, the incidence of CIN was higher in the iodinated contrast medium group (50\%, 1 of 2 patients) compared with the carbon dioxide group ( $0 \%, 0$ of 4 patients) [21]. In the single-center study, some patients felt mild transient abdominal discomfort immediately after carbon dioxide injection; however, none had nausea, vomiting, hypotension, narcosis, air contamination-related complications, or late complications related to carbon dioxide angiography. In a prospective multicenter trial of carbon dioxide angiography for PAD in CKD patients, the average doses of carbon dioxide and iodinated contrast medium were $281.4 \pm 155.8 \mathrm{~mL}$ and $15.0 \pm 18.1 \mathrm{~mL}$, respectively, and the incidence of CIN was 5.1\% [24]. In the multicenter trial, carbon dioxide-related complications occurred in $17.3 \%$ of the patients as well as leg pain (8.1\%), abdominal pain (6.1\%), diarrhea (1.0\%), and non-occlusive mesenteric ischemia (2.0\%).

In our case, we used the iodinated contrast medium in addition to carbon dioxide for below-the-knee arterial stenotic lesions in the kidney transplant patient due to the defocused image of below-the-knee stenotic lesions produced by carbon dioxide. Total PTAs with carbon dioxide $(200 \mathrm{~mL}$ per each PTA) were performed 3 times for PAD in the lower extremities and minimized the volumes of iodinated contrast medium $(15-30 \mathrm{~mL})$ after kidney transplantation. The patient had leg pain for 2 weeks only after the first PTA but did not have any major complications after these PTAs. One year later, the serum creatinine level was maintained, and the patient has shown no evidence of recurrence.

\section{Conclusions}

In a kidney transplant recipient with PAD, PTA with carbon dioxide was effective to minimize the volumes of iodinated contrast medium and prevent CIN incidence.

\section{Literature review}

There are 7 reports on intra-arterial treatment with carbon dioxide for PADs in kidney transplant recipients (Table 1) [25-31]. In 5 out of 7 reports, PTA with carbon dioxide for the stenosis of kidney transplant graft artery or iliac artery was performed [25-29]. In the remaining 2 reports, embolization with carbon dioxide for arteriovenous fistula occurring in kidney transplant graft after biopsy was performed [30, 31]. In these reports, mean volumes of carbon dioxide, iodinated, and gadolinium contrast medium were 20-114.6, 0-18.3, and $4-44 \mathrm{~mL}$, respectively. In 4 out of 7 reports, the mean serum creatinine levels were maintained after intra-arterial treatment $[25,28,29,31]$. In the only 1 report, the mean serum creatinine level elevated after intra-arterial treatment [26]. In the remaining 2 reports, there were no data for the mean serum creatinine levels before or after intra-arterial treatment [27, 30]. There were no descriptions of adverse events (except for kidney function) after intra-arterial treatment with carbon dioxide in all 7 reports. There are no reports on PTA with carbon dioxide for PAD in lower extremity after kidney transplantation. 


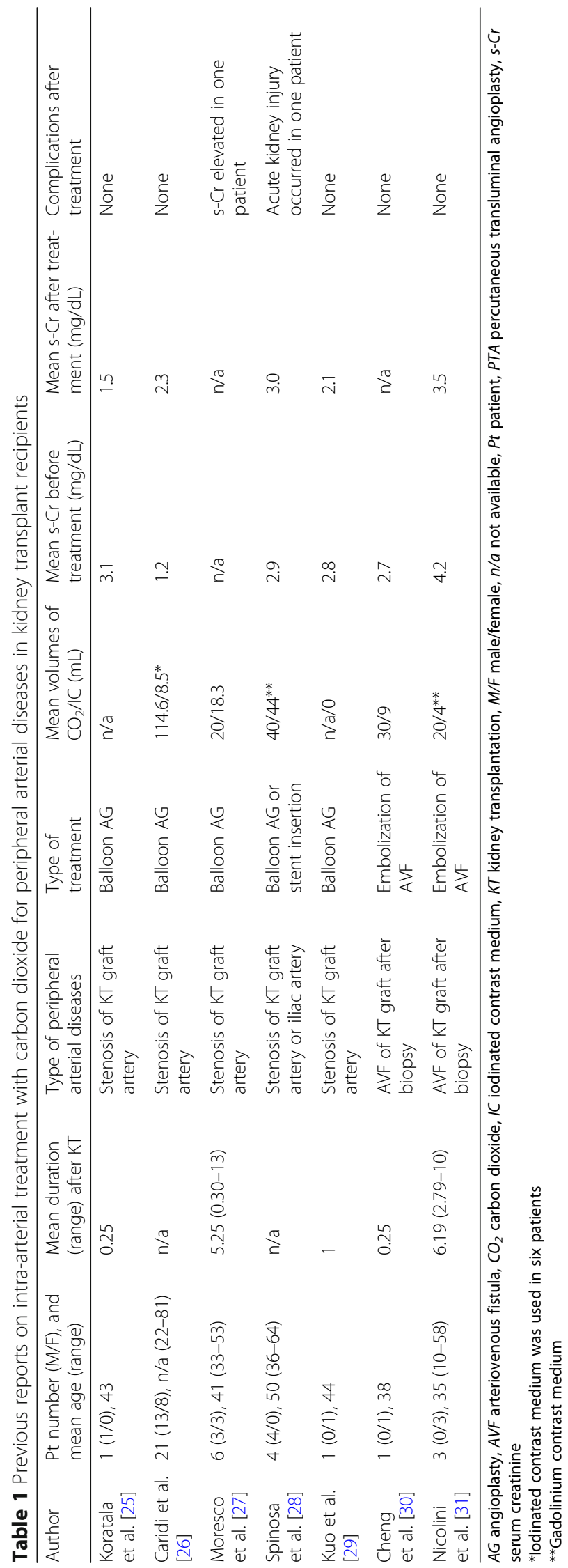




\section{Authors' contributions}

MB designed and wrote the manuscript. SM, SS, TS, MM, ST, HO, and HK provided intellectual content of critical importance to the work described. All authors read and approved the final manuscript.

\section{Funding}

No funding was obtained for this study.

\section{Availability of data and materials}

All data supporting our findings are contained within the manuscript.

\section{Ethics approval and consent to participate}

Not applicable.

\section{Consent for publication}

Written informed consent was obtained from the patients to publish this case report and any accompanying images. A copy of the written consent form is available for review by the editor of this journal.

\section{Competing interests}

The authors declare that they have no competing interests.

\section{Author details}

${ }^{1}$ Department of Artificial Organs, Akane-Foundation, Tsuchiya General Hospital, 3-30, Nakajimacho, Naka-ku, Hiroshima 730-8655, Japan. 2Department of Radiology, Akane-Foundation, Tsuchiya General Hospital, 3-30, Nakajimacho, Naka-ku, Hiroshima 730-8655, Japan. ${ }^{3}$ Department of Gastroenterological and Transplant Surgery, Applied Life Sciences, Institution of Biomedical \& Health Sciences, Hiroshima University, 1-2-3 Kasumi, Minami-ku, Hiroshima 734-8551, Japan.

Received: 31 October 2019 Accepted: 17 April 2020

Published online: 06 May 2020

\section{References}

1. Lamina C, Meisinger C, Heid IM, Lowel H, Rantner B, Koenig W, et al. Association of ankle-brachial index and plaques in the carotid and femoral arteries with cardiovascular events and total mortality in a population-based study with 13 years of follow-up. Eur Heart J. 2006;27(21):2580-7.

2. Saw J, Bhatt DL, Moliterno DJ, Brener SJ, Steinhubl SR, Lincoff AM, et al. The influence of peripheral arterial disease on outcomes: a pooled analysis of mortality in eight large randomized percutaneous coronary intervention trials. J Am Coll Cardiol. 2006:48(8):1567-72.

3. Menke A, Muntner P, Wildman RP, Dreisbach AW, Raggi P. Relation of borderline peripheral arterial disease to cardiovascular disease risk. Am J Cardiol. 2006;98(9):1226-30.

4. Higashi $Y$, Miyata $T$, Shigematsu $H$, Origasa $H$, Fujita M, Matsuo $H$, et al. Baseline characterization of Japanese peripheral arterial disease patientsanalysis of surveillance of cardiovascular events in antiplatelet-treated arteriosclerosis obliterans patients in Japan (SEASON). Circ J. 2016;80(3):71221.

5. Karthikeyan V, Karpinski J, Nair RC, Knoll G. The burden of chronic kidney disease in renal transplant recipients. Am J Transplant. 2004;4(2):262-9.

6. Mehran R, Nikolsky E. Contrast-induced nephropathy: definition, epidemiology, and patients at risk. Kidney Int Suppl. 2006;69(Suppl 100): S11-5.

7. Ahuja TS, Niaz N, Agraharkar M. Contrast-induced nephrotoxicity in renal allograft recipients. Clin Nephrol. 2000:54(1):11-4.

8. Haider M, Yessayan L, Venkat KK, Goggins M, Patel A, Karthikeyan V. Incidence of contrast-induced nephropathy in kidney transplant recipients. Transplant Proc. 2015;47(2):379-83.

9. Nash K, Hafeez A, Hou S. Hospital-acquired renal insufficiency. Am J Kidney Dis. 2002;39(5):930-6.

10. Neyra JA, Shah S, Mooney R, Jacobsen G, Yee J, Novak JE. Contrast-induced acute kidney injury following coronary angiography: a cohort study of hospitalized patients with or without chronic kidney disease. Nephrol Dial Transplant. 2013:28(6):1463-71.

11. James MT, Ghali WA, Knudtson ML, Ravani P, Tonelli M, Faris P, et al. Associations between acute kidney injury and cardiovascular and renal outcomes after coronary angiography. Circulation. 2011;123(4):409-16.
12. Wi J, Ko YG, Kim JS, Kim BK, Choi D, Ha JW, et al. Impact of contrast-induced acute kidney injury with transient or persistent renal dysfunction on longterm outcomes of patients with acute myocardial infarction undergoing percutaneous coronary intervention. Heart. 2011;97(21):1753-7.

13. Solomon RJ, Mehran R, Natarajan MK, Doucet S, Katholi RE, Staniloae CS, et al. Contrast-induced nephropathy and long-term adverse events: cause and effect? Clin J Am Soc Nephrol. 2009;4(7):1162-9.

14. Light JA, Perloff LJ, Etheredge EE, Hill G, Spees EK. Adverse effects of meglumine diatrizoate on renal function in the early post-transplant period. Transplantation. 1975;20(5):404-9.

15. Cigarroa RG, Lange RA, Williams RH, Hillis LD. Dosing of contrast material to prevent contrast nephropathy in patients with renal disease. Am J Med. 1989:86(6 Pt 1):649-52.

16. Gruberg L, Mintz GS, Mehran R, Gangas G, Lansky AJ, Kent KM, et al. The prognostic implications of further renal function deterioration within $48 \mathrm{~h}$ of interventional coronary procedures in patients with pre-existent chronic renal insufficiency. J Am Coll Cardiol. 2000;36(5):1542-8.

17. Briguori C, Manganelli F, Scarpato P, Elia PP, Golia B, Riviezzo G, et al. Acetylcysteine and contrast agent-associated nephrotoxicity. J Am Coll Cardiol. 2002:40(2):298-303.

18. Laskey WK, Jenkins C, Selzer F, Marroquin OC, Wilensky RL, Glaser R, et al. Volume-to-creatinine clearance ratio: a pharmacokinetically based risk factor for prediction of early creatinine increase after percutaneous coronary intervention. J Am Coll Cardiol. 2007:50(7):584-90.

19. Seeger JM, Self S, Harward TR, Flynn TC, Hawkins IF Jr. Carbon dioxide gas as an arterial contrast agent. Ann Surg. 1993;217(6):688-97 discussion 697-8.

20. Ohno I, Hayashi H, Aonuma K, Horio M, Kashihara N, Okada H, et al. Guidelines on the use of iodinated contrast media in patients with kidney disease 2012: digest version : JSN, JRS, and JCS Joint Working Group. Clin Exp Nephrol. 2013;17(4):441-79.

21. Chacko S, Joseph G, Thomson V, George P, George O, Danda D. Carbon dioxide angiography-guided renal-related interventions in patients with Takayasu arteritis and renal insufficiency. Cardiovasc Intervent Radiol. 2018; 41(7):998-1007.

22. Hawkins IF, Cho KJ, Caridi JG. Carbon dioxide in angiography to reduce the risk of contrast-induced nephropathy. Radiol Clin North Am. 2009;47(5):813-25 v-vi.

23. Diamantopoulos A, Patrone L, Santonocito S, Theodoulou I, Ilyas S, Dourado $\mathrm{R}$, et al. Carbon dioxide angiography during peripheral angioplasty procedures significantly reduces the risk of contrast-induced nephropathy in patients with chronic kidney disease. CVIR Endovasc. 2020;3(1):9.

24. Fujihara M, Kawasaki D, Shintani $Y$, Fukunaga M, Nakama T, Koshida R, et al. Endovascular therapy by $\mathrm{CO} 2$ angiography to prevent contrast-induced nephropathy in patients with chronic kidney disease: a prospective multicenter trial of $\mathrm{CO} 2$ angiography registry. Catheter Cardiovasc Interv. 2015;85(5):870-7.

25. Koratala A, Mena Gutierrez A, Chamarthi G, Zeng X, Alquadan KF. Two transplant renal arteries: one important lesson. Clin Case Rep. 2018;6(9):1909-10.

26. Caridi JG, Stavropoulos SW, Hawkins IF Jr. CO2 digital subtraction angiography for renal artery angioplasty in high-risk patients. AJR Am J Roentgenol. 1999;173(6):1551-6.

27. Moresco KP, Patel NH, Namyslowski Y, Shah H, Johnson MS, Trerotola SO. Carbon dioxide angiography of the transplanted kidney: technical considerations and imaging findings. AJR Am J Roentgenol. 1998;171(5):1271-6.

28. Spinosa DJ, Matsumoto AH, Angle JF, Hagspiel KD, Isaacs RB, McCullough CS, et al. Gadolinium-based contrast and carbon dioxide angiography to evaluate renal transplants for vascular causes of renal insufficiency and accelerated hypertension. J Vasc Interv Radiol. 1998;9(6):909-16.

29. Kuo PC, Petersen J, Semba C, Alfrey EJ, Dafoe DC. CO2 angiography--a technique for vascular imaging in renal allograft dysfunction. Transplantation. 1996;61(4):652-4.

30. Cheng PM, Van Allan RJ. Superior sensitivity of angiographic detection of arteriovenous fistula after biopsy in a renal allograft with CO2 compared with iodinated contrast medium. J Vasc Interv Radiol. 2006:17(12):1963-6.

31. Nicolini A, Ferraresso M, Lovaria A, Biondetti P, Raiteri M, Berardinelli L. Carbon dioxide as a valuable contrast agent for identifying iatrogenic arteriovenous fistulas in transplanted kidneys. Nephrol Dial Transplant. 2003; 18(10):2189-92.

\section{Publisher's Note}

Springer Nature remains neutral with regard to jurisdictional claims in published maps and institutional affiliations. 